\title{
A community-based sero-epidemiological study of hepatitis B infection in Lianyungang, China, 2010
}

\author{
Zhang Ting-lu, ${ }^{a}$ Xiao Zhi-ping, ${ }^{a}$ Ling Hong-yu, a Ge Chang-hong, ${ }^{a}$ Ying Liang, ${ }^{a}$ Ding Qiang, ${ }^{b}$ Xu Kai-ling, \\ Mao Yan-ming, ${ }^{a}$ Du Yue-he a and Zhu Ling-yang, ${ }^{\circ}$ \\ Correspondence to Zhang Ting-lu (e-mail: tlzhang66@gmail.com).
}

Introduction: The 2010 targets of the China Hepatitis B Prevention Programme were a prevalence of hepatitis B surface antigen (HBsAg) less than $1.0 \%$ for children less than five years old and less than $6.0 \%$ for the total population. This survey assessed the prevalence of Hepatitis B infection in Lianyungang, Jiangsu province, China in 2009-2010.

Methods: Multistage sampling was used with 2372 subjects among 17 selected villages. Blood specimen collection and testing by enzyme-linked immunosorbnet assay (ELISA) were completed using the following markers for hepatitis infection: HBsAg and antibody to HBsAg (anti-HBs); hepatitis B e antigen ( $\mathrm{HBeAg}$ ) and antibody to $\mathrm{HBeAg}$ (anti-HBe); and hepatitis B core antibody (total anti-HBc). The data were analyzed with Epi Info, version 3.3.2.

Results: The prevalence of HBsAg was 2.4\% (95\% Confidence Interval [Cl]: 1.8-3.0; Adjusted Prevalence [AP] 2.9\%); anti-HBs prevalence was 51.1\% (95\% Cl: 49.1-53.1; AP 49.2\%) and total anti-HBc prevalence was $41.7 \%$ (95\% Cl: 39.8-43.7; AP 45.5\%). The prevalence of HBsAg and total anti-HBc positivity increased from young to older age groups, yet the prevalence of anti-HBs positivity decreased from young to older age groups $(P<0.001$ for all). There was no difference in the prevalences of HBsAg and anti-HBs among females and males $(P=0.108$ and 0.089$)$, but females had a higher prevalence than males for total anti-HBc positivity $(P<0.001)$.

Discussion: This survey showed that in 2010 the prevalence of HBsAg among children aged less than five years was lower than the national target of $1.0 \%$ and that the prevalence of $\mathrm{HBsAg}$ for the total population was lower than the national target of $6.0 \%$.

\section{L}

ianyungang is one of the thirteen municipal cities in Jiangsu Province with four rural counties and four urban districts with a population of $4852400 .{ }^{1}$ In Lianyungang, the reported incidence of acute hepatitis B declined from 24.7 per 100000 in 1997 to 9.9 per 100000 in 2008, a decrease of $59.9 \%$. For those aged less than 15 years, the reported incidence of acute hepatitis B declined from 12.5 per 100000 in 1997 to 0.6 per 100000 in 2008, a decrease of $95.2 \%{ }^{2}$

According to the China Children Immunization Programme, a child must be vaccinated with three doses of the hepatitis $B$ vaccine before reaching 12 months of age. A child with the three-dose vaccination (first dose within 24 hours of birth, second at over one month and third at over six months; each dose is $5 \mu \mathrm{g}$ ) can be reported as immunized. The hepatitis $B$ vaccine became part of the immunization system in Lianyungang in 1992; free hepatitis B vaccination for newborn infants was carried out in 2001. From 2001 to 2009 the reported immunization coverage rate among children aged from 12 to 24 months in Lianyungang for each year was always more than $99 \%{ }^{3,4}$

China launched a hepatitis B catch-up immunization programme among children less than 15 years old in 2009. The target groups were those who had not completed a three-dose vaccination series or who had never received hepatitis B immunization. By 2010, Lianyungang had completed the catch-up immunization of hepatitis $B$ vaccine for children less than 15 years old.

A national serological investigation of hepatitis $B$ infection in China was conducted during the period 1992-1995 and showed that the prevalence for hepatitis B surface antigen ( $\mathrm{HBsAg}$ ) was $9.8 \%$; in Lianyungang it was $2 \%-7 \% .^{5}$

\footnotetext{
Lianyungang Municipal Center for Disease Control and Prevention, Lianyungang, Jiangsu Province, China.

Ganyu County Center for Disease Control and Prevention, Ganyu, Jiangsu Province, China.

Submitted: 3 March 2011 ; Published: 3 August 2012

doi: $10.5365 /$ wpsar.2011.2.1.008
} 
The China Hepatitis B Prevention Programme targets for 2010 were a prevalence of HBsAg lower than $1.0 \%$ for children less than five years old and a prevalence of $\mathrm{HBsAg}$ lower than $6.0 \%$ for the total population. ${ }^{5}$ As the reported incidence of hepatitis $B$ does not represent the prevalence for positive hepatitis $B$ markers, it was necessary to conduct a sero-epidemiological study to assess the prevalence of positive hepatitis $B$ markers among residents of Lianyungang.

This study aimed to determine the prevalence of positive hepatitis B markers in Lianyungang and assessed whether the national targets for 2010 were achieved. This sero-epidemiological investigation was carried out from 10 November 2009 to 15 April 2010.

\section{METHODS}

\section{Hepatitis B markers and test methods}

The hepatitis B markers tested in this study were hepatitis B surface antigen ( $\mathrm{HBsAg}$ ) and hepatitis B surface antibody (anti-HBs); hepatitis $B$ e antigen ( $\mathrm{HBeAg}$ ) and hepatitis $\mathrm{B}$ e antibody (anti-HBe); and hepatitis B core antibody (total anti-HBc).

HBsAg positivity indicates that the person is potentially infectious. Anti-HBs positivity indicates that the person obtained the antibody to HBsAg by vaccine or infection; anti-HBs is very useful to prevent hepatitis B infection or clear HBsAg. HBeAg positivity is associated with relatively high infectivity. Anti-HBe positivity indicates that the infectivity of hepatitis $B$ is lower than that in the period of $\mathrm{HBeAg}$ positivity. Total anti-HBc positivity indicates past or current hepatitis $B$ infection.

A $5 \mathrm{ml}$ blood specimen was collected from an arm vein with a vacuum blood tube $(2 \mathrm{ml}$ peripheral blood was collected from children under three years old). The blood serum was centrifuged in gel tubes within five hours of collection and then sent to the laboratory for testing.

Enzyme-linked immunosorbent assay (ELISA) was used to test the hepatitis $B$ markers with reagents. The HBsAg, anti-HBs, HBeAg and anti-HBe diagnostic reagents kits were from Beijing Kewei Clinical Diagnostic Reagents $\mathrm{Co}$, Ltd; the total anti-HBc diagnostic reagents kits were from Shanghai Kehua Bio-Engineering Co, Ltd.
The Model 680 microplate reader by BIO-RAD was used (Bio-Rad Laboratory, Inc, Hercules, California, USA). A sample was considered positive for $\mathrm{HBsAg}$, anti-HBs and $\mathrm{HBeAg}$ when the value of the optical density (OD) was $\geq 1$ (cutoff value); it was negative when the value of $\mathrm{OD}$ was $<1$ (cutoff value). A sample was considered positive for anti-HBe and total anti-HBc when the value of $\mathrm{OD}$ was $\leq 1$ (cutoff value); it was negative when the value of $O D$ was $>1$ (cutoff value). Blank tests were implemented at the same time.

$\mathrm{HBsAg}$, anti-HBs and total anti-HBc were tested in the first step to obtain the prevalence for $\mathrm{HBsAg}$ positivity, the prevalence for anti-HBs positivity and the prevalence for total anti-HBc positivity. We also tested for HBsAg-positive samples, $\mathrm{HBeAg}$ and anti-HBe. For those that were negative for $\mathrm{HBsAg}$ and/or anti-HBs we advised them to obtain the hepatitis B vaccine from clinics or hospitals.

\section{Sample}

The estimated sample size in this sero-epidemiological investigation was 2460, based on the prevalence of HBsAg carriers $(9.1 \%),{ }^{6}$ a relative precision of $10 \%$ and a confidence level of $90 \%$, allowing for $10 \%$ loss. The sample size was distributed to each of the four rural counties and four urban districts according to the proportion of population in Lianyungang, China in 2010 (Table 1).

Initially four rural counties were randomly selected - Ganyu, Donghai, Guanyun and Guannan. Within these four counties, stratified cluster samplings were done in three strata according to the cumulative hepatitis B cases identified by the China National Diseases Reporting System in these four counties during the period 1 January 2006 and 31 December 2008. The three strata were: more than 20 cases (high), 10-20 cases (middle) and less than 10 (low). One town per stratum was identified in each county by simple random sampling totalling 12 towns. Then one village was identified in each town in the same way, finally 12 villages were identified in three strata in four counties. The sample size was distributed to three villages in each county; for example, there was a sample size of 556 in Ganyu County; 556 divided by three resulted in a 185 sample size distributed to each village (Table 1 ). Simple random sampling was done in the urban districts (Lianyun, Xinpu, Haizhou and Kaifa) due to few communities and 
Table 1. Sample size distribution by country or district, Lianyungang, China, 2010

\begin{tabular}{|c|c|c|c|c|c|c|c|c|}
\hline \multirow{2}{*}{$\begin{array}{c}\text { County or } \\
\text { district }\end{array}$} & \multicolumn{2}{|c|}{ Population } & \multirow{2}{*}{$\begin{array}{l}\text { Sample } \\
\text { size }\end{array}$} & \multirow{2}{*}{$\begin{array}{c}\text { Selected } \\
\text { villages or } \\
\text { communities }\end{array}$} & \multirow{2}{*}{$\begin{array}{l}\text { Sample size } \\
\text { per village }\end{array}$} & \multirow{2}{*}{$\begin{array}{l}\text { Target no. of } \\
\text { households per } \\
\text { selected village }\end{array}$} & \multicolumn{2}{|c|}{ Tested sample } \\
\hline & $\mathbf{N}$ & $\%$ & & & & & $\mathbf{N}$ & $\%$ \\
\hline Ganyu & 1097618 & 22.6 & 556 & 3 & 185 & 62 & 531 & 22.4 \\
\hline Donghai & 1175967 & 24.2 & 596 & 3 & 199 & 66 & 586 & 24.7 \\
\hline Guanyun & 1104475 & 22.8 & 560 & 3 & 187 & 62 & 551 & 23.2 \\
\hline Guannan & 752266 & 15.5 & 381 & 3 & 127 & 42 & 372 & 15.7 \\
\hline Lianyun & 143630 & 3.0 & 73 & 1 & 73 & 24 & 70 & 3.0 \\
\hline Xinpu & 361212 & 7.4 & 183 & 2 & 92 & 31 & 181 & 6.7 \\
\hline Haizhou & 149703 & 3.1 & 76 & 1 & 76 & 25 & 71 & 3.0 \\
\hline Kaifa & 67529 & 1.4 & 34 & 1 & 34 & 11 & 30 & 1.3 \\
\hline Total & 4852400 & 100.0 & 2460 & 17 & 145 & 48 & 2372 & 100.0 \\
\hline
\end{tabular}

few hepatitis B cases, so five communities were selected in four districts according to the sample size (Table 1). A total of 17 villages or communities were selected.

Households were allocated a random number, and were then recruited based on the order of these random numbers. The selected persons must have lived with others in a housing unit for more than 12 months. It was estimated that there were three members in each household in this area, so the total estimated households were $820(2460 / 3)$.

All persons living within the sampled villages or community had a chance to be selected. However, persons who resided or worked in institutions were not eligible to participate in the investigation. An institution is a collective setting, for example, homes for the elderly or children, hostels, dormitories and military barracks, factories. All participants were advised they had the right to participate or withdraw from the investigation. Consent to participate and permission for a blood draw were obtained through a signature (or a fingerprint) on the consent form. The subjects were informed of their test results by telephone or mail or the participants could get their test results by calling the Lianyungang Center for Disease Control and Prevention. Prior to subject notification the results of HBsAg positivity were kept confidential to avoid discrimination when the test results were disclosed. All sensitive personal information collected during this investigation was kept confidential, including details of tattoos, sexual activity and orientation and drug use.

\section{Ethics}

We obtained approval from the Jiangsu Province Center for Disease Control and Prevention ethics committee before starting the sero-epidemiological investigation.

\section{Data collection and analysis}

A registration form was used to record the participant's name, age, address, telephone number, blood collection date, physical status and the results of the blood tests. Excel 2003 was used to set up the database and report the results of the blood tests. The data were analysed using Epi Info, version 3.3.2, including chi-squared tests and tests for trend which could take into account the study design. Prevalence figures were adjusted for age and reported as adjusted prevalence (AP).

\section{RESULTS}

\section{Participants}

The total number of participants who agreed to a blood draw was 2372, giving a response rate of $96.5 \%$. Non responders were either not interested in participating in the investigation or unavailable/unable to be found.

When compared to the Lianyungang population in 2009 , the proportion of survey participants in the 20-29 year age group was lower (16.1\% compared to $6.3 \%$ ) and the proportions of study participants 
Table 2. Comparison of study participants with the 2009 Lianyungang population by age group

\begin{tabular}{llrc}
\hline Age (years) & \multicolumn{2}{c}{ Study participants } & $\begin{array}{c}\text { Lianyungang } \\
\text { population, 2009 }\end{array}$ \\
\hline $0-4$ & $n$ & $\%$ & 5.4 \\
$5-9$ & 515 & 21.7 & 6.8 \\
$10-14$ & 397 & 16.7 & 9.9 \\
$15-19$ & 106 & 4.5 & 8.0 \\
$20-29$ & 149 & 6.3 & 16.0 \\
$30-39$ & 213 & 9.0 & 16.5 \\
$40-49$ & 292 & 12.3 & 13.6 \\
$50-59$ & 293 & 12.3 & 10.8 \\
Over 60 & 272 & 11.5 & 13.0 \\
$\boldsymbol{p}$ - value & & & $<\mathbf{0 . 0 0 1}$ \\
\hline & 2372 & 100.0 & 100.0 \\
\hline
\end{tabular}

in the 5-9 year and 10-14 year age groups were higher $(6.8 \%$ and $9.9 \%$ compared with $21.7 \%$ and $16.7 \%$ respectively) (Table 2). These differences were significantly different $(P<0.001)$.

\section{Prevalence of hepatitis B infection}

HBsAg prevalence among the 2372 survey participants was $2.4 \%(95 \% \mathrm{Cl}: 1.8-3.0)$; anti-HBs prevalence was $51.1 \%(95 \% \mathrm{Cl}: 49.1-53.1)$; total anti-HBC prevalence was $41.7 \%(95 \% \mathrm{Cl}$ : 39.8-43.7). When adjusted for age, the prevalence of $\mathrm{HBsAg}$, anti-HBs and total anti-HBc (AP) were $2.9 \%, 49.2 \%$ and $45.5 \%$, respectively (Table 3 ).

A total of $57 \mathrm{HBsAg-positive} \mathrm{specimens} \mathrm{were}$ tested again: 10 specimens were HBeAg-positive and anti-HBc-positive, 35 specimens were anti-HBe-positive and anti-HBc-positive, and another 12 specimens were both $\mathrm{HBeAg}$ and anti-HBe-negative and anti-HBcpositive. Three female specimens were HBsAg-positive and HBeAg-positive; two of the women were less than 20 years old, and one was over 60 years.

\section{Distribution by person}

The prevalence of $\mathrm{HBsAg}$ among children less than five years old was $0.7 \%(95 \% \mathrm{Cl}$ : $0.0-2.2$ (Table 3$)$. The prevalence of $\mathrm{HBsAg}$ among the $<15$-year-old age group was $0.8 \%(95 \% \mathrm{Cl}: 0.2-1.3)$, lower than that in other groups. The trend for prevalence of $\mathrm{HBsAg}$ and total anti-HBc positivity increased from the young to older age groups ( $P<0.001$ for both), yet the trend for prevalence of anti-HBs positivity decreased from young to older age groups $(P<0.001)$ (Table 3 ).

The prevalences of HBsAg and anti-HBs among females and males were no different $(P=0.108$ and 0.089). The prevalence for total anti-HBc positivity among females was $45.0 \%$ (95\% Cl: 42.3-47.7), significantly higher than that in males $(P<0.001)$ (Table 3).

Table 3. Seroprevalence of hepatitis B infection by age group and sex, Lianyungang, China, 2010

\begin{tabular}{|c|c|c|c|c|c|c|c|c|c|c|}
\hline & \multirow{2}{*}{$\mathbf{N}$} & \multicolumn{3}{|c|}{ HBsAg-positive } & \multicolumn{3}{|c|}{ Anti-HBs-positive } & \multicolumn{3}{|c|}{ Total anti-HBc-positive } \\
\hline & & $\mathbf{n}$ & $\%$ & $95 \% \mathrm{Cl}$ & $\mathrm{n}$ & $\%$ & $95 \% \mathrm{Cl}$ & $\mathrm{n}$ & $\%$ & $95 \% \mathrm{Cl}$ \\
\hline \multicolumn{11}{|c|}{ Age (years) } \\
\hline $0-4$ & 135 & 1 & 0.7 & $0.0-2.2$ & 80 & 59.3 & $51.0-67.5$ & 22 & 16.3 & $10.1-22.5$ \\
\hline $5-9$ & 515 & 5 & 1.0 & $0.1-1.8$ & 307 & 59.6 & $55.4-63.8$ & 132 & 25.6 & 22.9-29.4 \\
\hline $10-14$ & 397 & 2 & 0.5 & $0.0-1.2$ & 224 & 56.4 & $51.5-61.3$ & 114 & 28.7 & 24.3-33.2 \\
\hline 15-19 & 106 & 3 & 2.8 & $0.0-6.0$ & 59 & 55.7 & $46.2-65.1$ & 32 & 30.2 & $21.4-38.9$ \\
\hline $20-29$ & 149 & 6 & 4.0 & $0.9-7.2$ & 73 & 49.0 & $41.0-57.0$ & 63 & 42.3 & $34.3-50.2$ \\
\hline 30-39 & 213 & 4 & 1.9 & $0.1-3.7$ & 104 & 48.8 & $42.1-55.5$ & 104 & 48.8 & $42.1-55.5$ \\
\hline $40-49$ & 292 & 13 & 4.5 & $2.1-6.8$ & 134 & 45.9 & $40.2-51.6$ & 166 & 56.8 & $51.2-62.5$ \\
\hline $50-59$ & 293 & 12 & 4.1 & $1.8-6.4$ & 119 & 40.6 & $35.0-46.2$ & 183 & 62.5 & $56.9-68.0$ \\
\hline Over 60 & 272 & 11 & 4.0 & $1.7-6.4$ & 112 & 41.2 & $35.3-47.0$ & 174 & 64.0 & $58.3-69.7$ \\
\hline AP *(\%) & & \multicolumn{3}{|c|}{2.9} & \multicolumn{3}{|c|}{49.2} & \multicolumn{3}{|c|}{45.5} \\
\hline$p$-value & & \multicolumn{3}{|c|}{$<0.001$} & \multicolumn{3}{|c|}{$<0.001$} & \multicolumn{3}{|c|}{$<0.001$} \\
\hline \multicolumn{11}{|l|}{ Sex } \\
\hline Female & 1289 & 25 & 1.9 & $1.2-2.7$ & 638 & 49.5 & $46.8-52.2$ & 580 & 45.0 & $42.3-47.7$ \\
\hline Male & 1083 & 32 & 3.0 & $1.9-4.0$ & 574 & 53.0 & $50.0-56.0$ & 410 & 37.9 & $35.0-40.7$ \\
\hline$p$-value & & \multicolumn{3}{|c|}{0.108} & \multicolumn{3}{|c|}{0.089} & \multicolumn{3}{|c|}{$<0.001$} \\
\hline Total & 2372 & 57 & 2.4 & $1.8-3.0$ & 1212 & 51.1 & $49.1-53.1$ & 990 & 41.7 & $39.8-43.7$ \\
\hline
\end{tabular}

* AP - Adjusted prevalence for age using Lianyungang population in 2009. 
Table 4. Sero-prevalence of hepatitis B infection by county, Lianyungang, China, 2010

\begin{tabular}{|c|c|c|c|c|c|c|c|c|c|c|}
\hline \multirow{2}{*}{ Counties } & \multirow{2}{*}{$\mathbf{N}$} & \multicolumn{3}{|c|}{ HBsAg-positive } & \multicolumn{3}{|c|}{ Anti-HBs-positive } & \multicolumn{3}{|c|}{ Total anti-HBc-positive } \\
\hline & & $n$ & $\%$ & $95 \% \mathrm{Cl}$ & $n$ & $\%$ & $95 \% \mathrm{Cl}$ & $n$ & $\%$ & $95 \% \mathrm{Cl}$ \\
\hline \multicolumn{11}{|l|}{ Rural } \\
\hline Ganyu & 531 & 14 & 2.6 & $1.3-4.0$ & 284 & 53.5 & $49.2-57.7$ & 205 & 38.6 & $34.5-42.7$ \\
\hline Donghai & 586 & 6 & 1.0 & $0.2-1.8$ & 305 & 52.0 & $48.0-56.1$ & 213 & 36.3 & $32.5-40.2$ \\
\hline Guanyun & 551 & 20 & 3.6 & $2.1-5.2$ & 240 & 43.6 & $39.4-47.7$ & 264 & 47.9 & $43.7-52.1$ \\
\hline Guannan & 372 & 12 & 3.2 & $1.4-5.0$ & 233 & 62.6 & $57.7-67.6$ & 201 & 54.0 & $49.0-59.1$ \\
\hline \multicolumn{11}{|l|}{ Urban } \\
\hline Lianyun & 70 & 2 & 2.9 & $0.0-6.8$ & 31 & 44.3 & $32.6-55.9$ & 38 & 54.3 & $42.6-66.0$ \\
\hline Xinpu & 161 & 1 & 0.6 & $0.0-1.8$ & 61 & 37.9 & $30.4-45.4$ & 43 & 26.7 & $19.9-33.5$ \\
\hline Haizhou & 71 & 1 & 1.4 & $0.0-4.1$ & 48 & 67.6 & $56.7-78.5$ & 8 & 11.3 & $3.9-18.6$ \\
\hline Kaifa & 30 & 1 & 3.3 & $0.0-9.8$ & 10 & 33.3 & $16.5-50.2$ & 18 & 60.0 & $42.5-77.5$ \\
\hline$p$-value & & & 0.250 & & & 0.020 & & & $<0.00$ & \\
\hline Total & 2372 & 57 & 2.4 & $1.8-3.0$ & 1212 & 51.1 & $49.1-53.1$ & 990 & 41.7 & $39.8-43.7$ \\
\hline
\end{tabular}

\section{Distribution by place}

The county with the highest prevalence of HBsAg was Guanyun County at $3.6 \%(95 \% \mathrm{Cl}: 2.1-5.2)$; the lowest was Xinpu District at 0.6\% (95\% Cl: 0.0-1.8). The county with the highest prevalence of anti-HBs was Guannan County at $62.6 \%$ (95\% Cl: $57.7-67.6)$; the lowest was Kaifa District at $33.3 \%(95 \% \mathrm{Cl}$ : 16.5-50.2). The county with the highest prevalence for total anti-HBc positivity was Kaifa District at $60.0 \%$ (95\% Cl: 42.5-77.5); the lowest was Haizhou District at $11.3 \%(95 \% \mathrm{Cl}: 3.9-18.6)$ (Table 4).

There was no significant difference in the prevalence of $\mathrm{HBsAg}$ between all rural counties combined and all urban counties combined $(P=0.250)$, but there was a significant difference in the prevalence of antiHBs between all rural counties combined and all urban counties combined $(P=0.020)$ and a significant difference in the prevalence of total anti-HBc between all rural counties combined and all urban counties combined $(P<0.001)$ (Table 4).

\section{Distribution within rural counties by cumulative hepatitis B stratum}

Sampling in the four rural counties was completed using stratum based on cumulative hepatitis B case numbers. There was no significant difference in the prevalence of HBsAg between high, middle and low cumulative number of reported hepatitis $B$ case areas $(P=0.360)$; but there was a significant difference in the prevalence for anti-HBs positivity with the middle cumulative hepatitis $B$ case areas having a higher prevalence than the high and low stratum $(P<0.001)$. The prevalence of total anti-HBc positivity in high cumulative reported hepatitis B case area was $46.0 \%$ (95\% Cl: 42.3-49.6), which was not significantly higher than in middle and low cumulative reported hepatitis $B$ case areas $(P=0.175)($ Table 5).

\section{DISCUSSION}

This sero-epidemiological investigation of hepatitis B infection among a community-based population in

Table 5. Seroprevalence of hepatitis B infection in rural counties by cumulative reported hepatitis B cases strata, Lianyungang, China, 2010

\begin{tabular}{|c|c|c|c|c|c|c|c|c|c|c|}
\hline \multirow{2}{*}{ Stratum* } & \multirow{2}{*}{$\mathbf{N}$} & \multicolumn{3}{|c|}{ HBsAg-positive } & \multicolumn{3}{|c|}{ Anti-HBs-positive } & \multicolumn{3}{|c|}{ Total anti-Hbc-positive } \\
\hline & & $n$ & $\%$ & $95 \% \mathrm{Cl}$ & $n$ & $\%$ & $95 \% \mathrm{Cl}$ & $n$ & $\%$ & $95 \% \mathrm{Cl}$ \\
\hline High & 729 & 15 & 2.1 & $1.0-3.1$ & 346 & 47.5 & 43.8-51.1 & 335 & 46.0 & $42.3-49.6$ \\
\hline Middle & 646 & 21 & 3.3 & $1.9-4.6$ & 408 & 63.2 & $59.4-66.9$ & 274 & 42.4 & $38.6-46.2$ \\
\hline Low & 665 & 16 & 2.4 & $1.2-3.6$ & 308 & 46.3 & $42.5-50.1$ & 274 & 41.2 & $37.5-44.9$ \\
\hline$p$-value & & \multicolumn{3}{|c|}{0.360} & \multicolumn{3}{|c|}{$<0.001$} & \multicolumn{3}{|c|}{0.175} \\
\hline Total & 2040 & 52 & 2.5 & $1.9-3.2$ & 1062 & 52.1 & $49.9-54.2$ & 883 & 43.3 & 41.1-45.4 \\
\hline
\end{tabular}

*Stratum: high - more than 20 cases, middle - 10-20 cases and low - less than 10 cases. 
Lianyungang showed a prevalence of $\mathrm{HBsAg}$ of $2.4 \%$ (AP 2.9\%). This was lower than that in the 2002 national investigation $(9.1 \%){ }^{6}$ in the 2008 national serosurvey (weighted prevalence $7.2 \%)^{7}$ and in the 2006 Jiangsu investigation $(4.8 \%){ }^{8}$ The prevalence of $\mathrm{HBsAg}$ among children less than five years old was $0.7 \%$, which was lower than the $1.0 \%$ goal set by the China Hepatitis B Prevention Programme during the period 2006-2010. ${ }^{5}$ However, the confidence interval for this $0.7 \%$ was $0.0-2.2$, which is higher than the goal.

From 2001, free hepatitis B vaccination for newborn infants started in Lianyungang. This government-funded programme has made great achievements in the past 10 years. The reported coverage rate of hepatitis $B$ vaccination has increased to over $99 \%$ for children less than 10 years old..$^{3,4}$ The high coverage rate of hepatitis B vaccination has increased the prevalence for anti-HBs positivity among children less than 10 years old to over 59\% (387/650), higher than other age groups. The prevalence of anti-HBs for all survey participants was $51.1 \%$, which is higher than in the 2002 national investigation (39.90\%, AP $37.48 \%),{ }^{6}$ and similar to in the 2008 national serosurvey (weighted prevalence $50.1 \%)^{7}$ and in the 2006 Jiangsu investigation (50.34\%). ${ }^{8}$ The prevalence of anti-HBs for those aged 0-4 years was $59.3 \%$, which was lower than that reported in another Chinese serosurvey $(69.8 \%){ }^{9}$

The high coverage rate of hepatitis $B$ vaccination has lowered the hepatitis $B$ infection. The positivity for HBsAg among children less than 10 years old in this study was $0.9 \%(6 / 650)$, lower than that in other age groups; the positivity for total anti-HBc among children less than 10 years old was $23.7 \%$ (154/650), lower than that in the age groups over 20 years old, but the prevalence of total anti-HBc for all survey participants was $41.7 \%$, higher than that in the 2002 national investigation (20.2\%, AP 21.6\%), ${ }^{6}$ and that in the 2008 national serosurvey ${ }^{7}$ (weighted prevalence $34.1 \%$ ) but lower than that in the Jiangsu investigation $(49.8 \%){ }^{8}$

Analysis by cumulative hepatitis $B$ case stratum showed that the prevalence of anti-HBs positivity in the middle stratum (10 to 20 cases between 1 January 2006 and 31 December 2008) was higher than the high and low strata (more than 20 and less than 10 cases respectively, $P<0.001$ ). There was no significant difference in the prevalence of $\mathrm{HBsAg}$ between the three areas $(P=0.360)$ and for total anti-HBC $(P=0.175)$. Therefore, the cumulative hepatitis B cases from the China National Diseases Reporting System in four rural counties during 2006 and 2008 did not really represent the hepatitis B infection level in a certain area, specific to the prevalence of positive hepatitis $B$ markers.

From the coverage survey data for Lianyungang, we see high vaccine coverage over multiple years. This probably directly led to the decreasing burden of disease seen in the children tested in this study.

Our study had a response rate of $96 \%$; however, the age distribution of the study sample was significantly different to that of the Lianyungang population. During the investigation many senior students and migrant workers aged 20-40 years old were not at home, so these age groups were underrepresented. We did adjust prevalence estimates for age to account for this.

\section{CONCLUSION}

This sero-epidemiological investigation showed the prevalence of $\mathrm{HBsAg}$ among children less than five years old had been controlled to less than $1.0 \%$; the prevalence of $\mathrm{HBsAg}$ for all the population, at $2.4 \%$ was less than $6 \%$. These data indicate that the hepatitis $\mathrm{B}$ immunization programmes has been effective in Lianyungang in the past few years.

\section{Conflicts of interests}

None declared.

Funding

This sero-epidemiological investigation of hepatitis B infection was sponsored by the Lianyungang Health Bureau.

\section{Acknowledgements}

We thank Mr Yang Guang-ze for early programming assistance, all county and municipal Center for Disease Control and Prevention laboratory staff for blood specimen collection and testing, clinic staff for organizing the participants and arranging the investigation. 


\section{References:}

1. Lianyungang Statistical Yearbook, 2009, 53-57.

2. Zhang, Ting-lu et al. Incidence Trend of Hepatitis B in Lianyungang, 1997-2008. Journal of Preventive Medicine Information, 2010, 7:507-510.

3. Jiang HB. Evaluation on Immunization Coverage Surveillance during 2001-2004 in Lianyungang [in Chinese]. Modern Preventive Medicine, 2006, 8:1461-1462.

4. Jiang $\mathrm{BH}$. Evaluation on Immunization Coverage Surveillance during 2005-2009 in Lianyungang (awaiting publication in Modern Preventive Medicine).

5. China Hepatitis B Prevention Programme during 2006-2010. Beijing, Ministry of Health of the People's Republic of China, 2006, No. 39.
6. Liang XF et al. A study on the sero-epidemiology of hepatitis $B$ in Chinese population aged over 3-years-old [in Chinese]. Chinese Journal of Epidemiology, 2005, 9:655-658. pmid: 16471211

7. Liang $X$ et al. Epidemiological serosurvey of hepatitis $B$ in China-declining HBV prevalence due to hepatitis B vaccination. Vaccine, 2009, 27:6550-6557. doi:10.1016/j.vaccine.2009. 08.048 pmid: 19729084

8. Chun-yu LI. Investigation of the general people for Hepatitis B Virus, evaluation of the reagent and a primary probe into the mini-pool test. A dissertation submitted to Southeast University for the Academic Degree of Master of Medicine, 2006:7-14.

9. Cui FQ, Bi SL, Zhang Y. Combination profiles of hepatitis B marks for Chinese in serosurvey in 2006 [in Chinese]. Zhongguo Yi Miao He Mian Yi, 2009, 15:294-299. pmid:20077724 